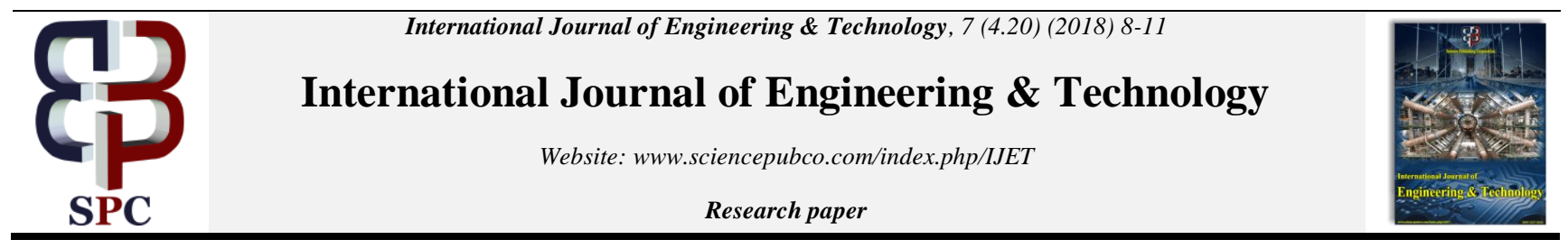

\title{
Traffic aware channel assignment with node stability in wireless mesh networks
}

\author{
D.JasmineDavid $^{1^{*}}$, V. Jegathesan ${ }^{2}$ \\ ${ }^{I}$ Department of ElectricalTechnology, Karunya University, Coimbatore. \\ *Corresponding author E-mail:jasmine@karunya.edu
}

\begin{abstract}
In a wireless network the most challenging issue is Channel assignment. The channel assignment problem is codependent with the routing problem. We need to compute again the channel assignment as and when the traffic pattern changes. Anyways, previously followed channel assignment algorithms will assign channels from scratch. It will end up with an entirely dissimilar configuration of nodes; in turn it will disturb the action of the particular network. It takes little time to create links and to establish new channels. This time is significant in assigning links for wireless networks. This leads to channel reassignment. This algorithm considers the existing channel assignment and tries to go along with the new stream of traffic flow design in the finest possible way by changing the channel on a restricted number of radios. In order to provide node stability, we used entropy function. In this paper, we demonstrate a channel reallocation algorithm with node permanency and appraise its performance by using NS2. Experimental outcomes show that the node stability can progress the performance of network when compared with the earlier methods.
\end{abstract}

Keywords: Wireless mesh networks, channel assignment, reassignment, node permanency.

\section{Introduction}

From the beginning, wireless mesh networks (WMNs) have fascinated lots of interest from research communal due to its simplicity and cost effectiveness. It has attracted industries also since it covers the urban areas deprived of the wired infrastructure making it cheap to device. One of the main issues that attract researchers is allocating channels with multiple radios for a wireless mesh network. Configuring nodes with multiple radios is very common nowadays. Hence, it's genuine to reduce the interference and to increase throughput by meticulously allocating channels to radios.

The allocation of channels is based on of the routing problem. By any means if the nodes are using the same channel, it's necessary that they have to share the capability of the channel. The allocation of channel should not disturb the link bandwidth. Therefore, always the channel allocation should go with routing to achieve better results. Recent literature addressed the channel allocation and routing problems distinctly. A communal way is to resolve the routing first and then to assign channel.

The allocation of channel depends on the rate of flows, it ought to be reassigned depends upon the inconsistency of the load. If reassignment occurs often, that way of allocating channel is undesirable. Implementing the new channel assignment will change the network topology. The connectivity of the network breaks if the channel is changed very often. Also, it takes more time to swing to new frequency. The routing algorithm will take a while to judge the unavailability of the already assigned link and it has to admit the new link to prevent the routing oscillations. Once a radio is chosen to a new channel, the routing algorithm will take a while to admit the links in the new channel. As a consequence, packet losses will occur and it may increase the retransmission thereby decreasing the network performance. Maximum of the existing channel allocation algorithms offer suboptimal solution. Our channel reallocation with node stability algorithm treasures an improved result. The rest of the paper is organized as follows, in section 2 presents the related work. Section 3 presents node stability-based channel reassignment algorithm. Section 4 deals the performance of the proposed work. In section 5 conclusion and future work is discussed.

\section{Related Work}

In WMN the channel allocation is examined in the collected works in recent times. There are countless schemes to reduce the interference. These schemes represent only the channel allocation problem. But not considered the routing problem. In [1], the aim is to catch a channel allocation that reduces the magnitude of congestion domain. The algorithm offered in [2] considers traffic produced by mesh clients. In [3], super imposed codes are used to find the interference less channel allocation. A rank based channel allocation MesTiC [4] is used. In [5], centralized and distributed algorithms are offered to reduce the interfering link. A distributed channel allocation process and routing presented in [6]. In [7] distributed protocol for channel allocation and routing for mesh networks is proposed. 
Routing based traffic schemes projected in [8]. In [9], explanation for channel allocation and routing is established. The probability of a agreed set of flows between source and destination is verified in [10]. In [11], channel allocation, preparation, and routing algorithm are presented.

Complete channel allocation algorithms are given as survey in [12] for WMN. A traffic-aware channel allocation for wireless sensor networks is presented in [13]. To detect the interference between nodes and congestion less time division multiple access based detection is projected in [14].

All these papers consider only the channel allocation, but if the flow of the traffic changes the reconfiguration procedure is not stated. The method in [15] adopts the presence of a link layer harmonization between nodes and establishes the data communication at diverse time slots without congestion. This algorithm reconfigures the channel allocation and the link scheduling as a significance of a variation in the traffic matrix. In [16], considers the variation in traffic due to the handoffs of mesh client while reassigning channel. This paper offers an improved form of the channel reallocation scheme that improves reconfiguration in transmission rates, better description of the link significances and extra precise and complete performance evaluation.

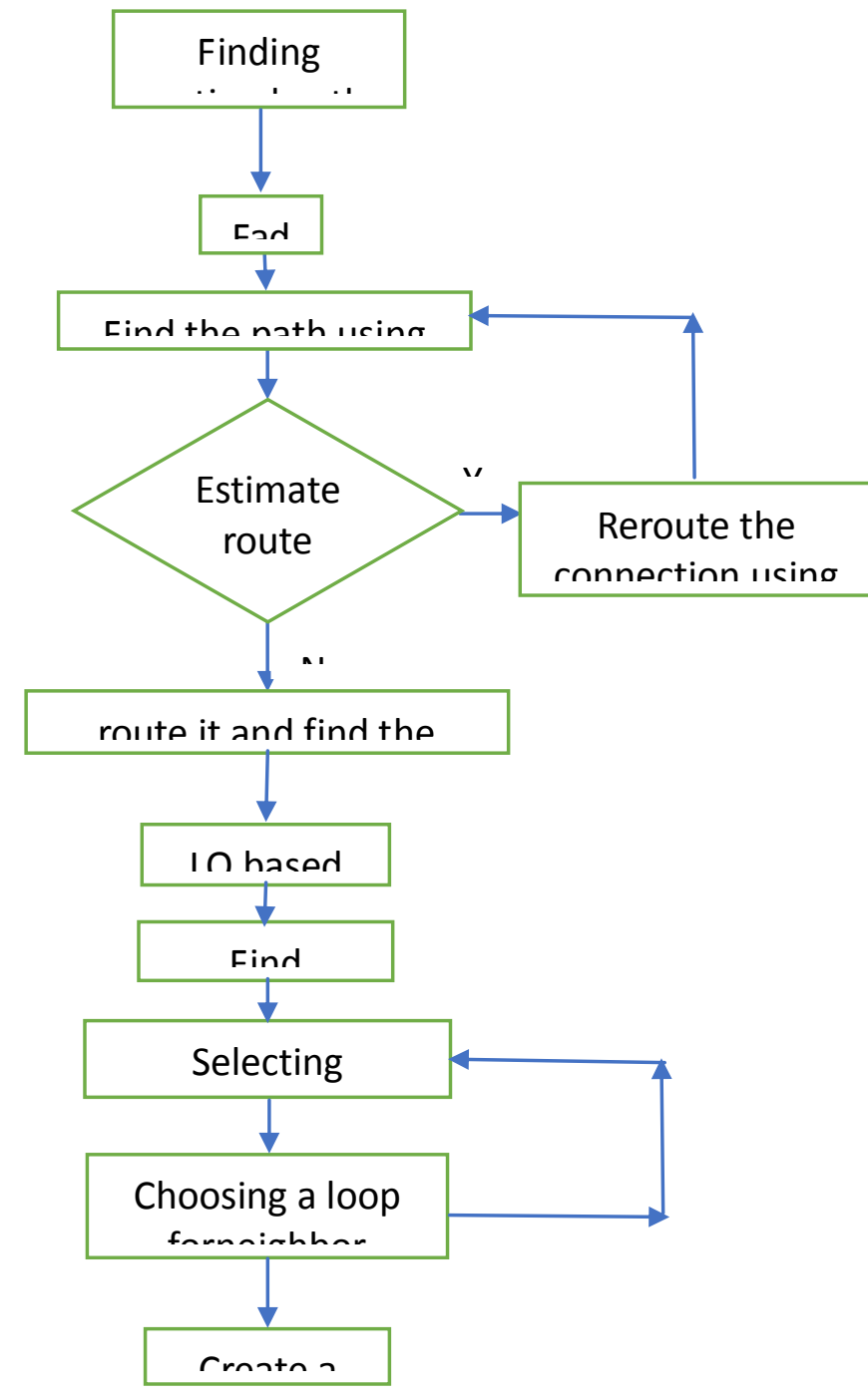

Fig. 1 Methodology Flow Chart
There are numerous factors that affect the signal loss or data packets in wireless networks. One among them is fading, in our paper we are concentrating on this factor and try to diminish the loss.

Initially, find a shortest path in which the traffic traces are less and now check for the route disconnection. If there is no disconnection, it can follow that path if not then data packets have to choose another path.

This selecting of another path is done using minimum variation channel route reassignment (MVCRR) algorithm. This algorithm makes the data packets to find the link connectivity and at the same time looks for the channel where the traffic is very low. Thus, channels are reassigned.

Now, to maintain node permanence we adopt 2 other algorithms namely 1) Stability index algorithm 2) Loop free forwarding algorithm. The quality of the link is based on the SNR value and using that value node permanency is determined.

Once node permanency is found out then depends on the previous history link is selected. But when the link is chosen that way there is a chance of forming an infinite loop (Let us consider a node A, node $\mathrm{B}$, node $\mathrm{C}$ are neighbors first data packet moves from node $\mathrm{A}$ to node B since it is neighboring node. Similarly, from node B to node $\mathrm{C}$, since node $\mathrm{A}$ is also neighbor to node $\mathrm{C}$ there is a chance for data packet to move to node A again) resulting to form an infinite loop. To eradicate this problem, we use loop free forwarding algorithm.

Finally, a path is created such that there is minimum information loss and with a faster movement. Nodes sharing the same channel at the same time cause inter and intra flow interferences.

The uncertainty of the network can be the main reason for packet losses and rise network latency. The factors that cause the network uncertainty are interferences, routing protocols, network topology and traffic patterns, congestion, route flapping and effects of exploration.

The proposed algorithm reduces the extreme utilization of the assigned channels. While minimalize the entire utilization of the available channel the accessibility of multiple transmission rate is also exploited

Thus, our proposed channel reassignment algorithm regulates both the channel and the transmission rate on selected links to further decrease the extreme total utilization.

Stability index of a link should be calculated and stores in the routing table of a router before describing the permanency of a node. By using the history of the previous link quality and present quality the link's stability index is calculated. If the metric of the link quality is smaller than the threshold it is considered as the acceptable link. The link that oscillates between acceptable and unacceptable values will not be considered for transmission of packets since it causes instability. The projected algorithm calculates the stability index of a link by using link oscillations among the acceptable and unacceptable, link persistence, and link quality development/worsening. This scheme adds either the acceptable or unacceptable links in the routing table. For unacceptable link the stability index value is considered as 0 whereas for actable link it is 1. At the end of each transmission period the link quality is checked. If there is no change in the quality of the link, the stability index is incremented by 1 . If there is any improvement in the quality of the link when compared with the previous value, then the stability index will be incremented by 2 .

\section{Simulation Study}

An example is executed in the NS-2 software with the following parameters, the values are mentioned below 


\section{Simulation Parameters Values}

\begin{tabular}{|lc|}
\hline Network Area & $1000 \mathrm{mx} 1000 \mathrm{~m}$ \\
\hline Link Data Rate & $11 \mathrm{Mbps}$ \\
\hline Frequency & $9.14 \mathrm{e}+08 \mathrm{~Hz}$ \\
\hline Traffic Type & CBR (UDP) \\
\hline Network Load & $1000-3000 \mathrm{kbps}$ \\
\hline Propagation Model & Two Ray Ground \\
\hline Simulation time & $125 \mathrm{~s}$ \\
\hline Goodput & 20579.6 \\
\hline Throughput & $108068 \mathrm{bps}$ \\
\hline Packet Delivery ratio & 99.7101 \\
\hline Jitter & 0.038860 \\
\hline Delay & $0.209776 \mathrm{~s}$ \\
\hline Packets received & 2752 \\
\hline
\end{tabular}

\section{Plotted Graphs:}

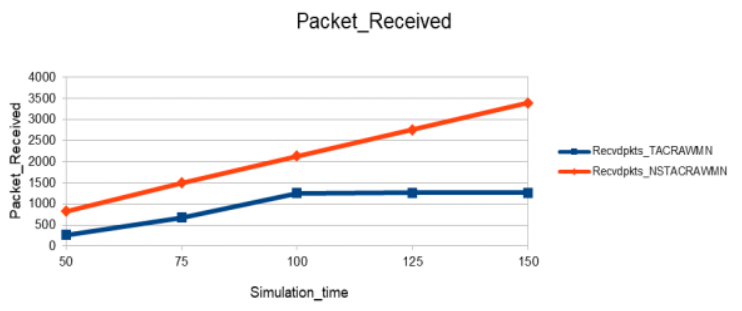

Packet_Delivery_Ratio

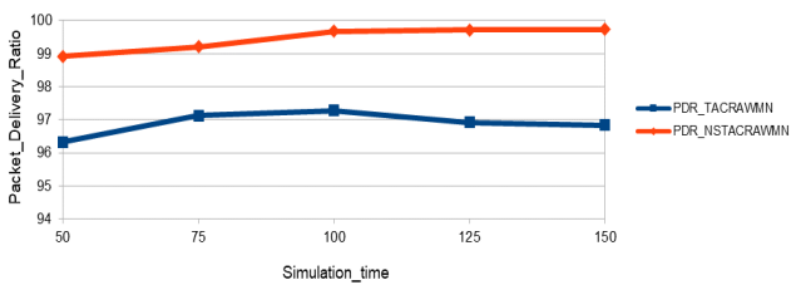

The ratio of packets that are successfully delivered to a destination compared to the number of packets that have been sent out by the sender.

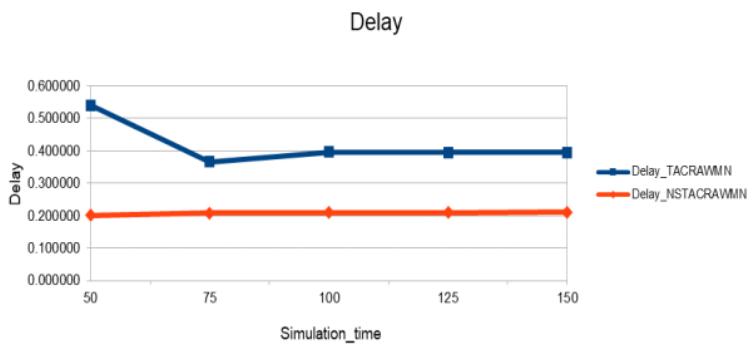

Network delay is an important design and performance characteristic of network. The delay of a network is the time taken by a packet to reach the destination. It can be measured in fractions of seconds.

Packet_Dropped

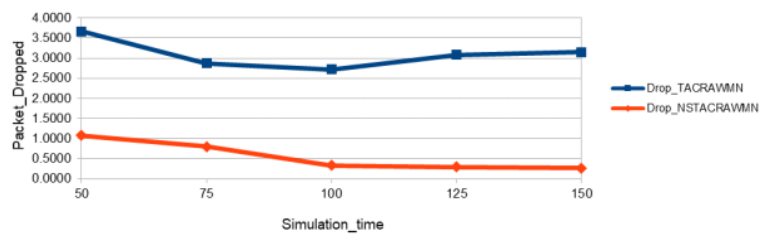

If more than one packet travels across the network to reach the destination packet loss happens. It is initiated either by errors in data transmission or by network congestion. Packet loss is measured as a percentage of packets lost with respect to packets sent.

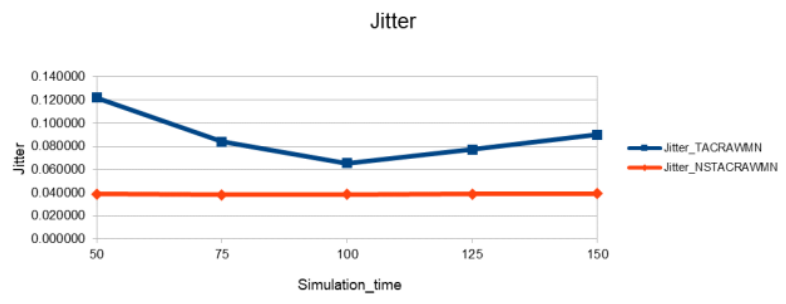

Jitter

is the difference in packet transmits delay produced by queue up and congestion that effects on the path through the network. In general, sophisticated stages of jitter occur on either slow or severely congested links.

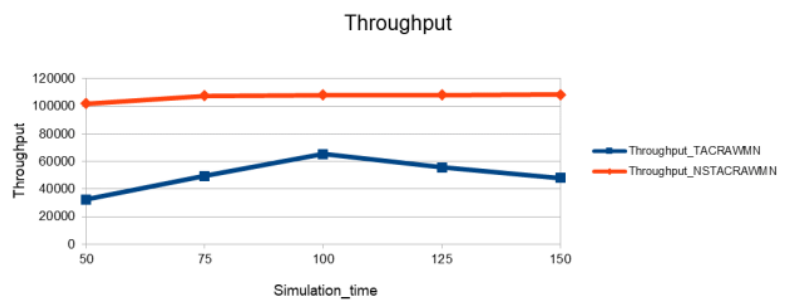

Throughput is the amount of fruitful message delivery over a communication channel. Throughput is typically measured in bits per second, and sometimes in data packets per second or data packets per time slot.

\section{Conclusion}

Minimum variation channel route reassignment algorithm minimizes the congestion domain utilization by considering the flow of traffic. Network stability is also included with this algorithm to increase the network performance. It cares the real-time communication over wireless networks. This algorithm considers the existing channel assignment and tries to go along with the new stream of traffic flow design in the finest possible way by changing the channel on a restricted number of radios. In order to provide node stability, we used entropy function. In this paper, we demonstrate a channel reallocation algorithm with node permanency and appraise its performance by using NS2. Experimental outcomes show that the node stability can progress the performance of network when compared with the earlier methods.

\section{References}

[1] J. Tang, G. Xue, and W. Zhang, "Interference-Aware Topology Control and QoS Routing in Multi-Channel Wireless Meshetworks," Proc. ACM MobiHoc, pp. 68-77, 2005.

[2] K.N. Ramachandran, E.M. Belding, K.C. Almeroth, and M.M. Buddhikot, "Interference-Aware Channel Assignment in Multi- Radio Wireless Mesh Networks," Proc. IEEE INFOCOM, 2006.

[3] K. Xing, X. Cheng, L. Ma, and Q. Liang, "Superimposed Code Based Channel Assignment in Multi-Radio Multi-Channel Wireless Mesh Networks," Proc. ACM MobiCom, pp. 15-26, 2007.

[4] H. Skalli, S. Ghosh, S. Das, L. Lenzini, and M. Conti, "Channel Assignment Strategies for Multiradio Wireless Mesh Networks: Issues and Solutions," IEEE Comm. Magazine, vol. 45, no. 11, pp. 86-95, Nov. 2007.

[5] A. Subramanian, H. Gupta, S.R. Das, and J. Cao, "Minimum Interference Channel Assignment in Multi-Radio Wireless Mesh 
Networks," IEEE Trans. Mobile Computing, vol. 7, no. 12, pp. 1459 1473, Dec. 2008.

[6] A. Raniwala and T. Chiueh, "Architecture and Algorithms for an IEEE 802.11-Based Multi-Channel Wireless Mesh Network," Proc.IEEE INFOCOM, vol. 3, pp. 2223-2234, 2005.

[7] A. Dhananjay, H. Zhang, J. Li, and L. Subramanian, "Practical,Distributed Channel Assignment and Routing in DualRadio Mesh Networks," Proc. ACM SIGCOMM, pp. 99-110, Aug. 2009.

[8] A. Raniwala, K. Gopalan, and T. Chiueh, "Centralized Channel Assignment and Routing Algorithms for Multi-Channel Wireless Mesh Networks," ACM Mobile Computing and Comm. Rev., vol. 8, no. 2, pp. 50-65, Apr. 2004.

[9] M. Alicherry, R. Bhatia, and E. Li, "Joint Channel Assignment and Routing for Throughput Optimization in Multiradio Wireless Mesh Networks," IEEE J. Selected Areas in Comm., vol. 24, no. 11,pp. 1960-1971, Nov. 2006.

[10] M. Kodialam and T. Nandagopal, "Characterizing the Capacity Region in Multi-Radio Multi-Channel Wireless Mesh Networks,"Proc. ACM MobiCom, pp. 73-87, 2005.

[11] X. Lin and S. Rasool, "A Distributed Joint Channel-Assignment, Scheduling and Routing Algorithm for Multi-Channel Ad-Hoc Wireless Networks," Proc. IEEE INFOCOM, pp. 1118-1126, 2007.

[12] J. Crichigno, M.-Y. Wu, and W. Shu, "Protocols and Architectures for Channel Assignment in Wireless Mesh Networks," Ad Hoc Networks, vol. 6, no. 7, pp. 1051-1077, 2008.

[13] Y. Wu, M. Keally, G. Zhou, and W. Mao, "Traffic-Aware Channel Assignment in Wireless Sensor Networks," Proc. Int'l Conf. Wireless Algorithms, Systems, and Applications (WASA), pp. 479- 488, 2009.

[14] G. Zhou, T. He, J. Stankovic, and T. Abdelzaher, "RID: Radio Interference Detection in Wireless Sensor Networks," Proc. IEEE INFOCOM, vol. 2, pp. 891-901, Mar. 2005.

[15] Akyildiz, I.F., Wang, X., Wang, W., 2005. Wireless mesh networks: a survey. ComputerNetwork. 47 (4), 445-487

[16] An, B., Lee, J.-.S., Kim, N.-.S., 2009." An Entropy-based Routing Protocol using Mobility in Mobile Ad-hoc Wireless Sensor Networks". In: Proceedings of the 11th IIEEE international Conference on Advanced Communication Technology, pp. 949-952.

[17]Aydin, I., Jaikaeo, C., Shen, C.C., 2005. Quorum-based match-making services forwireless mesh networks. In Proceedings of the International Conference on Wireless

And Mobile Computing, Networking And Communications, pp. 377-382. 